\title{
Design and Implementation of Stock-Aware Shelves using Pervasive Computing
}

\author{
Neeraj Anturkar \\ Savitribai Phule \\ Pune University \\ Pune Maharashtra, India
}

\author{
Ashwin Adhav \\ Savitribai Phule \\ Pune University \\ Pune Maharashtra, India
}

\author{
Prajkta Rangnekar \\ Savitribai Phule \\ Pune University \\ Pune Maharashtra, India
}

\author{
Pratik Gokhale \\ Savitribai Phule \\ Pune University \\ Pune Maharashtra, India
}

\author{
Bhakti Patil \\ Savitribai Phule \\ Pune University \\ Pune Maharashtra, India
}

\begin{abstract}
Pervasive computing also called ubiquitous computing is nothing but embedding microprocessors in everyday objects so they can communicate information. With the advancements in Internet technologies, a new trend in the era of ubiquity is being realized. Today, whenever we go out to buy grocery we may forget to buy any particular, which may cause inconvenience. This problem can be solved by using Pervasive Computing principles.

Smart phones and wearable devices are being used to make the world around us reachable, inter-actable and to keep us updated. Utilizing sensor and smartphone technology we target to embed a mind in kitchen which interacts with user and automatically generates To-buy list and alerts depending upon the need of a particular item. Towards such a perspective, there is the need to continuously collect, elaborate, and present data, possibly deriving from smart objects. This can be achieved by the use of Sensor Networks, consisting of raspberry pi 3 development board along with HX711 load sensor interface to sense the weight of particular quantity and send it witlessly over the network. This raspberry pi 3 can be programmed in Python to serve our purpose.
\end{abstract}

\section{General Terms}

HX711, RC522, Raspberry Pi, Monitoring stock

\section{Keywords}

Internet of Things (IoT), Pervasive Computing, Stock Awareness, Sensor Network, Load sensing

\section{INTRODUCTION}

In embedded systems the current worldwide trend is ubiquitous computing. It enables communication between everyday objects by embedding microprocessors in everyday objects to make our life simpler. This particular idea revolves around sensing the load of the shelves thereby detecting the level of stocks and transmitting it to user's mobile device. We extend this concept to solve everyday problems like forgetting purchase of a particular grocery item.

Devices like smartphones and wearables keep us reachable, interactable and updated to the everyday events happening around the world. We target to embed a mind in shelves, utilizing sensor and smartphone technology an automated to buy list is generated. To implement such a system, there is the need to continuously fetch, elaborate, and analyze data deriving from smart objects. This is achieved by the use of Sensor Networks, consisting of raspberry pi 3 development kit along with HX711 load sensor interface. HX711 senses the exact weight of particular container and seamlessly syncs it wirelessly over the network. Using python programming we can customise raspberry pi 3 to serve our purpose. Using machine learning and predictive analysis, users usage statistics can be analyzed to predict his needs.

\subsection{Problem Statement}

To develop a pervasive system to which senses the level of stocks and shares this information with users mobile device to generate alerts.

\section{LITERATURE SURVEY}

Issues that arise in retail store like out of stock are a major concern for Consumer Packaged Goods and Retail organizations. They lose billions of dollars every year. If items of interest are not available on the shelves, most of the customers buy from somewhere else and others buy a different brand. [1]Describes how the shelves at a retail store can be made smarter such that they can raise alerts to restock themselves. Alerts to workers in retail shop can skip manual checks and alerts to CPG companies can accelerate refill if retailers do not maintain the right quantity or restock within Service Level Agreement (SLA). Using load sensors have been the most common practice yet it has not succeeded as anything else on the shelves still meant available. Thus the challenges we face are to track not only the quantity but also the right brand, right variant in the right position with right visibility to shoppers on the right shelf. Internet of Things can help make this thing (shelves) smarter to required extent. The authors have enabled shelves to take its own selfie using a simple camera and then the images processing algorithms takes care of performing required analytics and generate alerts with the person responsible. This solution scores higher over other hardware and capital intensive IoT solutions being worked upon.

[2] In pervasive retail, Radio Frequency Identification (RFID)-enabled smart shelves are becoming common place. Real-time information about the items stock and location is provided by these devices, that being fact, few efforts have been made to reliably detect human interaction with the items. Novel approach is presented by author on real-time humanobject interaction detection. This approach is based on RFID using supervised machine learning techniques. 
[3] The surge in the number of internet users and how the internet of things phenomena is affecting human lives. Home automation has immense impact and usage. The future of computing deals with embedding the sensors etc with the everyday use devices, the encompass several domains such as e-Governance, health care, Transportation, Waste Management, Food Supply Chains, and Energy and Utilization. Different labs such as iDorm, Smart home Monitoring etc. Also ambient intelligence is planted to at home to get the detail information about the people living.

[4] In pervasive retail, Radio Frequency Identification (RFID)-enabled smart shelves are becoming common place. Real-time information about the items stock and location is provided by these devices, that being fact, few efforts have been made to reliably detect human interaction with the items. Novel approach is presented by author on real-time humanobject interaction detection. That approach is based on RFID using supervised machine learning techniques. After analyzing specific features of RFID human interaction with the smart shelf has been studied and more than $84 \%$ efficiency is noted.

[5] WSN is of utter importance in IoT. WSNs bring IoT applications richer capabilities for both sensing and actuation. WSN solutions already have a wide range of applications, and research and technology advances continuously expand their application field. This trend also increases their use in IoT applications for versatile low-cost data acquisition and actuation.

[7] Kitchen is a very essential place of a home and cooking is one of the day to day activities. The usual difficulty in a kitchen during cooking is finding the items that are exhausted. The extensive use of automated systems indicates the demand of the household devices to be smart and automated to support us in our daily activities. The kitchen is one ideal place where automation at various levels can be done.

[8] An exploratory research project named "Smart Tupperware" conducted in Personal Task Assistants (PTA) is examining both devices and user interfaces for personal augmentation systems that can assist in such tasks as repeatedly maintaining kitchen foodstuffs inventory and integrating it with a shopping list application. Through sensor built in kitchen containers and various mnemonic methods, the system measures the fill status of the containers and maintains a distributed database that is uploaded to a personal device that is network-connected and user aware.

\section{SYSTEM ARCHITECTURE}

The overall system design consists of following major modules:
(a) Hardware
(b) Server
(c) Mobile Device

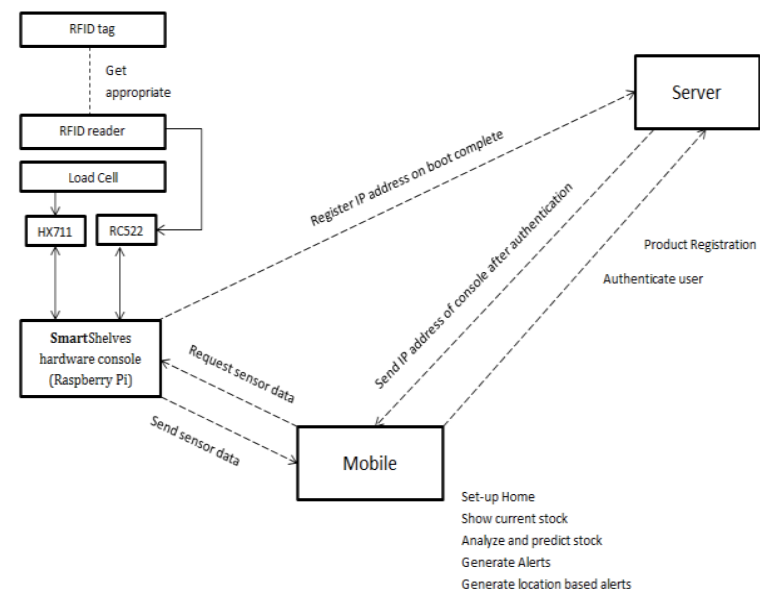

Figure 4.1: System Architecture

The Hardware module uniquely identifies the containers using RFID and RC522, senses load using load sensor and HX711 and transmits data to mobile device when requested

The Server module is responsible to authenticate the user, gets IP address from the hardware and sends it to users mobile device after authentication. It also stores a database of hardware devices.

The Mobile Device Requests hardware for sensor data, Performs operations on data, makes it available to the user, Generates alerts on low stock, Generates shop location based alerts, Generates "To-Buy "list.

\section{EVALUATION OF SYSTEM 4.1 Advantages}

The system provides access to authorized personals only. It generates alerts based on exhaustion of stocks. This system is real time and user friendly.

\subsection{Disadvantages}

The seamless working of the product largely depends upon the network signal quality. Calibration of the load sensors can be a deployment time overhead. Also the system is expensive to scale up.

\subsection{Applications}

This idea can be used extensively in industry where the raw materials are supplied just in time, hospitals where the level of medicine stock is critical or hotel management industry where physically monitoring the stocks is tedious.

\section{CONCLUSION}

Thus the system provides easy monitoring of stock inventory by using load sensors, RFID, Raspberry Pi and a mobile device application providing significant advantages like availability of real time sensor data on users mobile device, easy to use and set up application, implicit analysis of fetched data and generating alerts based on the same, prediction of data using machine learning etc. Although, this approach comes with a few disadvantages like overhead of calibrating load sensors or expensive to scale up, the idea of sensing quantity of stock using load sensors and making it implicitly available to the users can be extensively use in manufacturing industry where stocks of raw material are tedious to monitor or if unexpected exhaustion of a particular raw material halts the manufacturing process. In this approach the 
devices are networked which is an attribute of poor level ubiquity. It can further be improved to be choreographed.

\section{REFERENCES}

[1] Rakesh Satapathy, Srikanth Prahlad, Vijay Kaulgud, "Smart Shelfie Internet of Shelves For higher on-shelf availability", 2015 IEEE Region 10 Symposium

[2]Joan Melia-Segu and Rafael Pous, "Human-object Interaction Reasoning using RFID-enabled Smart Shelf',2014 International Conference on the Internet Of Things

[3]Sean Dieter Tebje Kelly, Nagender Kumar Suryadevara, and Subhas Chandra Mukhopadhyay, Fellow, IEEE, "Towards the Implementation of IoT for Envi- ronmental Condition Monitoring in Homes",IEEE SENSORS JOURNAL, VOL. 13, NO. 10, OCTOBER 2013

[4]Paolo Bellavista, Senior Member, IEEE, Giuseppe Cardone, Member, IEEE, An- tonio Corradi, Member, IEEE, and Luca Foschini, Member, IEEE, "Convergence of MANET and WSN in IoT Urban Scenarios ", IEEE
SENSORS JOURNAL, VOL. 13, NO. 10, OCTOBER 2013

[5]Mihai T. Lazarescu, “Design of a WSN Platform for LongTerm Environmental Monitoring for IoT Applications ",IEEE , MAY 2013

[6]Carla R. Medeiros, Jorge R. Costa, Member, IEEE, and Carlos A. Fernandes, Senior Member, IEEE,"RFID Smart Shelf With Confined Detection Volume at UHF ",IEEE ANTENNAS AND WIRELESS PROPAGATION LETTERS, VOL. 7, 2008

[7]Karuppiah Pal Amutha, Chidambaram Sethukkarasi, Raja Pitchiah, "Smart Kitchen Cabinet for Aware Home ",SMART 2012 : The First International Con- ference on Smart Systems, Devices and Technologies

[8]Richard Voyles, Jaewook Bae, Bret Smith, David Kusuma and Ledu Nguyen,"Smart Tupperware: Active Containers for Kitchen Automation"SICE Annual Conference 2008

[9]"Imagine IoT", openSAP MOOC, September 2016 